\title{
Emergence and expansion of cosmic space as due to $M 0$-branes
}

\author{
Alireza Sepehri ${ }^{1,2, a}$, Mohammad Reza Setare ${ }^{3, b}$, Salvatore Capozziello $^{4,5,6, c}$ \\ ${ }^{1}$ Faculty of Physics, Shahid Bahonar University, P.O. Box 76175, Kerman, Iran \\ ${ }^{2}$ Research Institute for Astronomy and Astrophysics of Maragha (RIAAM), P.O. Box 55134-441, Maragha, Iran \\ ${ }^{3}$ Department of Science, University of Kurdistan, Campus of Bijar, Bijar, Iran \\ ${ }^{4}$ Dipartimento di Fisica, Universita di Napoli Federico II, 80126 Naples, Italy \\ 5 INFN Sez. di Napoli, Compl. Univ. di Monte S. Angelo, Edificio G, 80126 Naples, Italy \\ ${ }^{6}$ Gran Sasso Science Institute (INFN), Viale F. Crispi, 7, 67100 L'Aquila, Italy
}

Received: 13 June 2015 / Accepted: 14 December 2015 / Published online: 29 December 2015

(C) The Author(s) 2015. This article is published with open access at Springerlink.com

\begin{abstract}
Recently, Padmanabhan (arXiv:1206.4916 [hepth]) discussed that the difference between the number of degrees of freedom on the boundary surface and the number of degrees of freedom in a bulk region causes the accelerated expansion of the universe. The main question arising is: what is the origin of this inequality between the surface degrees of freedom and the bulk degrees of freedom? We answer this question in $M$-theory. In our model, first $M 0$-branes are compactified on one circle and $N D 0$-branes are created. Then $N D 0$-branes join each other, grow, and form one $D 5$-branes. Next, the D5-brane is compactified on two circles and our universe's $D 3$-brane, two $D 1$-branes and some extra energies are produced. After that, one of the $D 1$-branes, which is closer to the universe's brane, gives its energy into it, and this leads to an increase in the difference between the numbers of degrees of freedom and the occurring inflation era. With the disappearance of this D1-brane, the number of degrees of freedom of boundary surface and bulk region become equal and inflation ends. At this stage, extra energies that are produced due to the compactification cause an expansion of the universe and deceleration epoch. Finally, another $D 1$-brane dissolves in our universe's brane, leads to an inequality between degrees of freedom, and there occurs a new phase of acceleration.
\end{abstract}

\section{Introduction}

About 3 years ago, Padmanabhan suggested that the accelerated expansion of the universe is due to the difference between the surface degrees of freedom on the holographic

\footnotetext{
a e-mail: alireza.sepehri@uk.ac.ir

be-mail: rezakord@ipm.ir

c e-mail: capozziello@na.infn.it
}

horizon and the bulk degrees of freedom through a simple equation $\Delta V=\triangle t\left(N_{\text {sur }}-N_{\text {bulk }}\right)$ where $V$ is the Hubble volume in Planck units and $t$ is the cosmic time in Planck units [1]. Up to date, many discussions have appeared on the Padmanabhan proposal [2-8]. For example, in one paper, with the help of this idea, the Friedmann equations of an $(n+1)$ dimensional Friedmann-Robertson-Walker universe corresponding to general relativity, Gauss-Bonnet gravity, and Lovelock gravity have been obtained [2]. In other research, the idea of treating the cosmic space as an emergent process has been applied to brane cosmology, scalar-tensor cosmology, and $f(R)$ gravity, and the corresponding cosmological equations in these theories have been derived [3]. In another investigation, using a suggestion due to Padmanabhan, the author obtained the Friedmann equations of the universe not only in four dimensional space-time and Einstein gravity, but also in higher dimensional space-time and other gravities like Gauss-Bonnet and Lovelock gravity with any spatial curvature [4]. Some other authors have extended the evolution equation according to Padmanabhan's idea to give the Friedmann equation in the nonflat universe corresponding to $k= \pm 1$ by taking into account the invariant volume surrounded by the apparent horizon [5]. In another scenario, the authors showed that applying Padmanabhan's conjecture to non-Einstein gravity cases one encounters serious difficulties and it has to be heavily modified to get the Friedmann equation [6]. In another paper, the author applied the derived equations of the universe in the Padmanabhan model with the corrected entropy-area law that follows from a generalized uncertainty principle (GUP) and obtained modified Friedmann equations due to the GUP [7]. In more recent research, the Padmanabhan idea has been constructed in a BIonic system and it was shown that all degrees of freedom inside and outside the universe are controlled by the evolutions of BIon in extra dimension and tend to the degrees of freedom of 
a black $F$-string in string theory [8]. The BIon is a configuration of two branes which are connected by a wormhole [9-13].

Now, the main question arises: what is the origin of the difference between the number of degrees of freedom on the boundary surface and the one in a bulk? To answer this question, we use the method in [13]. In that paper, $k$ fundamental strings decay to $N$ pairs of $M 0$-branes. Then these branes are glued to each other and form an M3, an anti-M3 and a wormhole between them. Our universe is located on one of these branes and interact with another brane via the wormhole. Extending this idea, we propose a new model which allows one to construct our universe from $M 0$-branes in $M$-theory. In this proposal, first, we will compactify $M 0$-branes on one circle and obtain the relevant action for $N D 0$-branes. Then we will show that these $D 0$-branes may join to each other and make a transition to a $D 5$-brane. Next, we will compactify this D5-brane on two circles and derive the relevant actions for one D3-brane, two D1-branes, and some extra energies. Our universe is located on this D3-brane and two D1-branes are the main causes of the inequality between the number of degrees freedom on the surface horizon and in a bulk, and the occurring inflation and late-time acceleration. Also, extra energies are responsible for a deceleration epoch.

The outline of the paper is as follows. In Sect. 2, we will construct a $D 0$-brane from an $M 0$-brane and consider the relation between their algebras. We also show that $D 0$-branes can join each other and form a D5-brane. Then we will compactify the $D 5$-brane on two circles and obtain one D3-brane and two D1-branes. In Sect. 3, we will show that the D1brane which is closer to the D3-brane dissolves in it and leads to inflation. Also, in this section, we will argue that another D1-brane is the main cause of the second phase of acceleration and inequality between the number of degrees of freedom on the holographic surface and the one in the bulk. The last section is devoted to a summary and our conclusion.

\section{The birth of the universe in $M$-theory}

In this section, we will show that the origin of the universe is in M0-branes. Recently, some authors have proposed an action based on Lie 3-algebras to describe M2branes [14-18]. Some other authors have considered the case of infinite dimensional Lie 3-algebras based on the Nambu-Poisson structure of three dimensional manifolds. They argued that the model contains self-dual 2-form gauge fields in six dimensions, and the result may be interpreted as the M5-brane world-volume action [19]. Extending these methods, we propose a new model which allows one to construct our universe's brane from $M 0$-branes. To this end, first, we obtain the relevant action for $N M 0$-branes by replacing the Nambu-Poisson structure of two dimensional manifolds in $D$-branes by the structure of a three dimensional one. In a second stage, we will compactify them on one circle and derive the action for $N D 0$-branes. We show that $N D 0$ branes join to each other, grow, and form a D5-brane. Then this brane is compactified on two circles and our universe's brane and two D1-branes are created.

First, we introduce the Born-Infeld action for the M0brane by replacing the two dimensional Nambu-Poisson bracket [20-26] in the action of $D p$-branes by a three dimensional Nambu-Poisson bracket [14-19] and applying a Lie 3-algebra [13]:

$$
\begin{aligned}
S_{M 0}= & T_{M 0} \int \mathrm{d} t \operatorname{Tr}\left(\Sigma _ { M , N , L = 0 } ^ { 1 0 } \left\langle\left[X^{M}, X^{N}, X^{L}\right],\right.\right. \\
& {\left.\left.\left[X^{M}, X^{N}, X^{L}\right]\right\rangle\right) }
\end{aligned}
$$

where $X^{M}=X_{\alpha}^{M} T^{\alpha}$ and

$$
\begin{aligned}
{\left[T^{\alpha}, T^{\beta}, T^{\gamma}\right] } & =f_{\eta}^{\alpha \beta \gamma} T^{\eta}, \\
\left\langle T^{\alpha}, T^{\beta}\right\rangle & =h^{\alpha \beta} \\
{\left[X^{M}, X^{N}, X^{L}\right] } & =\left[X_{\alpha}^{M} T^{\alpha}, X_{\beta}^{N} T^{\beta}, X_{\gamma}^{L} T^{\gamma}\right], \\
\left\langle X^{M}, X^{M}\right\rangle & =X_{\alpha}^{M} X_{\beta}^{M}\left\langle T^{\alpha}, T^{\beta}\right\rangle,
\end{aligned}
$$

where $X^{M}(i=1,3, \ldots, 10)$ 's refer to transverse scalars to the $M 0$-brane. One can show that by compactifying $M$ theory on a circle of radius $R$, the above action transits to a ten dimensional action for the D0-brane [24,26]. To this end, we apply the method in $[27,28]$ and define $\left\langle X^{10}\right\rangle=\frac{R}{l_{p}^{3 / 2}}$ where $l_{p}$ is the Planck length. We obtain [13]

$$
\begin{aligned}
& S_{M 0}=-T_{M 0} \int \operatorname{dt} \operatorname{Tr}\left(\Sigma _ { M , N , L = 0 } ^ { 1 0 } \left\langle\left[X^{M}, X^{N}, X^{L}\right],\right.\right. \\
& \left.\left.\left[X^{M}, X^{N}, X^{L}\right]\right\rangle\right) \\
& =-T_{M 0} \int \mathrm{dt} \operatorname{Tr}\left(\Sigma_{M, N, L, E, F, G=0}^{10} \varepsilon_{M N L D} \varepsilon_{E F G}^{D}\right. \\
& \left.X^{M} X^{N} X^{L} X^{E} X^{F} X^{G}\right) \\
& =-6 T_{M 0} \int \operatorname{dt} \operatorname{Tr}\left(\Sigma_{M, N, E, F=0}^{9} \varepsilon_{M N 10 D} \varepsilon_{E F 10}^{D}\right. \\
& \left.\times X^{M} X^{N} X^{10} X^{E} X^{F} X^{10}\right) \\
& -6 T_{M 0} \int \mathrm{dt} \Sigma_{M, N, L, E, F, G=0, \neq 10}^{9} \varepsilon_{M N L D} \varepsilon_{E F G}^{D} \\
& \times X^{M} X^{N} X^{L} X^{E} X^{F} X^{G} \\
& =-6 T_{M 0}\left(\frac{R^{2}}{l_{p}^{3}}\right) \int \mathrm{dt} \operatorname{Tr}\left(\Sigma_{M, N, E, F=0}^{9} \varepsilon_{M N 10 D^{\varepsilon_{E}}}^{D}\right. \\
& \left.\times X^{M} X^{N} X^{E} X^{F}\right) \\
& -6 T_{M 0} \int \mathrm{dt} \Sigma_{M, N, L, E, F, G=0, \neq 10}^{9} \varepsilon_{M N L D} \varepsilon_{E F G}^{D} \\
& \times X^{M} X^{N} X^{L} X^{E} X^{F} X^{G}
\end{aligned}
$$




$$
\begin{aligned}
= & -6 T_{M 0}\left(\frac{R^{2}}{l_{p}^{3}}\right) \int \mathrm{dt} \operatorname{Tr}\left(\Sigma_{M, N=0}^{9}\left[X^{M}, X^{N}\right]^{2}\right) \\
& -6 T_{M 0} \int \mathrm{dt} \Sigma_{M, N, L, E, F, G=0, \neq 10^{9} \varepsilon_{M N L D} \varepsilon_{E F G}^{D}} \\
& \times X^{M} X^{N} X^{L} X^{E} X^{F} X^{G} \\
= & S_{D 0}-6 T_{M 0} \int \mathrm{dt} \Sigma_{M, N, L, E, F, G=0, \neq 10^{9} \varepsilon_{M N L D} \varepsilon_{E F G}^{D}} \\
& \times X^{M} X^{N} X^{L} X^{E} X^{F} X^{G} S_{D 0}+V_{\text {Extra }, 1}
\end{aligned}
$$

where $V_{\text {Extra, } 1}=-6 T_{M 0} \int \mathrm{dt} \Sigma_{M, N, L, E, F, G=0}^{9} \varepsilon_{M N L D} \varepsilon_{E F G}^{D}$ $X^{M} X^{N} X^{L} X^{E} X^{F} X^{G}$. Also, $T_{M 0}$ and $T_{D 0}$ denote the tensions of $M 0$ and $D 0$, respectively, and $T_{D 0}=6 T_{M 0}\left(\frac{R^{2}}{l_{p}^{3}}\right)=$ $\frac{1}{g_{s} l_{s}}$ where $g_{s}$ and $l_{s}$ are the string coupling and string length, respectively. Clearly, the action (3) for compactified $M 0$ branes is equal to the sum of the relevant actions for the D0-brane [13,20,21,23-26],

$S_{D 0}=-T_{D 0} \int \mathrm{dt} \operatorname{Tr}\left(\Sigma_{m=0}^{9}\left[X^{m}, X^{n}\right]^{2}\right)$

and some extra energies that are produced due to compactification. Now, we can construct other $D p$-branes from $D 0$ branes by substituting the following rules [13,20,21,23-26]:

$$
\begin{aligned}
& \Sigma_{a=0}^{p} \Sigma_{m=0}^{9} \rightarrow \frac{1}{\left(2 \pi l_{s}\right)^{p}} \int \mathrm{d}^{p+1} \sigma \Sigma_{m=p+1}^{9} \Sigma_{a=0}^{p} \quad \lambda=2 \pi l_{s}^{2}, \\
& {\left[X^{a}, X^{i}\right]=i \lambda \partial_{a} X^{i} \quad\left[X^{a}, X^{b}\right]=i \lambda^{2} F^{a b}} \\
& i, j=p+1, \ldots, 9 \quad a, b=0,1, \ldots, p \\
& \quad m, n=0,1, \ldots, 9
\end{aligned}
$$

in the action (4) and doing some mathematical calculations [13]:

$$
\begin{aligned}
S_{D p}= & \Sigma_{a=0}^{p} S_{D 0}=-\Sigma_{a=0}^{p} T_{D 0} \int \mathrm{dt} \operatorname{Tr}\left(\Sigma_{m=0}^{9}\left[X^{m}, X^{n}\right]^{2}\right) \\
= & -T_{D p} \int \mathrm{d}^{p+1} \sigma \operatorname{Tr}\left(\Sigma_{a, b=0}^{p} \Sigma_{i, j=p+1}^{9}\right. \\
& \left.\times\left\{\partial_{a} X^{i} \partial_{b} X^{i}-\frac{1}{2 \lambda^{2}}\left[X^{i}, X^{j}\right]^{2}+\frac{\lambda^{2}}{4}\left(F_{a b}\right)^{2}\right\}\right),
\end{aligned}
$$

which is in agreement with results of [20,21,23-26] for the $D 1, D 3$, and $D 5$-branes. Here $T_{D p}=\frac{T_{D 0}}{\left(2 \pi l_{s}\right)^{p}}$ is the brane tension, $l_{s}$ is the string length, $g_{s}$ is the string coupling and $F_{a b}$ is the field strength. To compactify this $D p$-brane on one circle, we need to replace some gauge fields by some scalar fields and use the following laws [13,19-21,23-28]:

$$
\begin{aligned}
& T_{D p} \int \mathrm{d}^{p+1} \sigma \operatorname{Tr} \Sigma_{a, b=0}^{p} \Sigma_{i, j=p+1}^{9} \\
& \rightarrow T_{D(p-1)} \int \mathrm{d}^{p} \sigma \operatorname{Tr} \Sigma_{a, b=0}^{p-1} \Sigma_{i, j=p}^{9}
\end{aligned}
$$

$$
\begin{gathered}
+T_{D(1)} \int \mathrm{d}^{2} \sigma \operatorname{Tr} \Sigma_{a, b=0}^{1} \Sigma_{i, j=2}^{9} \\
i \lambda F_{a p} \rightarrow \partial_{a} X^{p} \quad i \partial_{p} X^{i} \rightarrow \frac{1}{\lambda}\left[X^{p}, X^{i}\right] .
\end{gathered}
$$

Replacing these equations in the action (6) and doing some algebra, we obtain

$$
\begin{aligned}
S_{D p}= & -T_{D p} \int \mathrm{d}^{p+1} \sigma \operatorname{Tr}\left(\Sigma _ { a , b = 0 } ^ { p } \Sigma _ { i , j = p + 1 } ^ { 9 } \left\{\partial_{a} X^{i} \partial_{b} X^{i}\right.\right. \\
& \left.\left.-\frac{1}{2 \lambda^{2}}\left[X^{i}, X^{j}\right]^{2}+\frac{\lambda^{2}}{4}\left(F_{a b}\right)^{2}\right\}\right) \\
= & -T_{D(p-1)} \int \mathrm{d}^{p} \sigma \operatorname{Tr}\left(\Sigma _ { a , b = 0 } ^ { p - 1 } \Sigma _ { i , j = p } ^ { 9 } \left\{\partial_{a} X^{i} \partial_{b} X^{i}\right.\right. \\
& \left.\left.-\frac{1}{2 \lambda^{2}}\left[X^{i}, X^{j}\right]^{2}+\frac{\lambda^{2}}{4}\left(F_{a b}\right)^{2}\right\}\right) \\
& -T_{D 1} \int \mathrm{d}^{2} \sigma \operatorname{Tr}\left(\Sigma _ { a , b = 0 } ^ { 1 } \Sigma _ { i , j = 2 } ^ { 9 } \left\{\partial_{a} X^{i} \partial_{b} X^{i}\right.\right. \\
& \left.\left.-\frac{1}{2 \lambda^{2}}\left[X^{i}, X^{j}\right]^{2}+\frac{\lambda^{2}}{4}\left(F_{a b}\right)^{2}\right\}\right)-V_{\text {separation }} \\
\rightarrow & S_{D p}=S_{D(p-1)}+S_{D 1}-V_{\text {separation }} \\
\rightarrow & S_{D p}+V_{\text {separation }}=S_{D(p-1)}+S_{D 1} .
\end{aligned}
$$

This equation shows that we need some extra energies that are applied for separating $D 1$-brane from $D p$-brane, $V_{\text {separation }}=-T_{D 1} \int \mathrm{d}^{2} \sigma \operatorname{Tr} \Sigma_{i, j=2}^{9} \frac{1}{2 \lambda^{2}}\left[X^{i}, X^{j}\right]^{2}$. To supply this energy, we make use of extra energies that are produced due to compactification of $M 0$-branes. To this aim, we compactify $V_{\text {Extra }}$ on the circle on defining $\left\langle X^{p}\right\rangle=\sqrt{\frac{T_{D 1}}{12 T_{M 0} \lambda^{2}}}$ :

$$
\begin{aligned}
V_{\text {Extra }, 1}= & -6 T_{M 0} \int \mathrm{dt} \Sigma_{M, N, L, E, F, G=0}^{9} \varepsilon_{M N L D} \varepsilon_{E F G}^{D} \\
& \times X^{M} X^{N} X^{L} X^{E} X^{F} X^{G} \\
= & -6 T_{M 0} \int \mathrm{dt} \operatorname{Tr}\left(\Sigma_{M, N, E, F=0}^{9} \varepsilon_{M N p D} \varepsilon_{E F p}^{D}\right. \\
& \left.\times X^{M} X^{N} X^{p} X^{E} X^{F} X^{p}\right) \\
& -6 T_{M 0} \int \mathrm{dt} \Sigma_{M, N, L, E, F, G=0, \neq p}^{8} \varepsilon_{M N L D} \varepsilon_{E F G}^{D} \\
& \times X^{M} X^{N} X^{L} X^{E} X^{F} X^{G} \\
= & -6 T_{M 0}\left(\frac{T_{D 1}}{12 T_{M 0} \lambda^{2}}\right) \int \mathrm{dt} \operatorname{Tr}\left(\Sigma_{M, N, E, F=0}^{9} \varepsilon_{M N p D}\right. \\
& \times \varepsilon_{E F p}^{D} X^{M} X^{N} X^{E} X^{F} \\
& -6 T_{M 0} \int \mathrm{dt} \Sigma_{M, N, L, E, F, G=0, \neq p}^{8} \varepsilon_{M N L D} \varepsilon_{E F G}^{D} \\
& \times X^{M} X^{N} X^{L} X^{E} X^{F} X^{G} \\
= & -\left(\frac{T_{D 1}}{2 \lambda^{2}}\right) \int \mathrm{dt} \operatorname{Tr}\left(\Sigma_{M, N=0}^{9}\left[X^{M}, X^{N}\right]^{2}\right) \\
& -6 T_{M 0} \int \mathrm{dt} \Sigma_{M, N, L, E, F, G=0, \neq p}^{8} \varepsilon_{M N L D} \varepsilon_{E F G}^{D} \\
& \times X^{M} X^{N} X^{L} X^{E} X^{F} X^{G}
\end{aligned}
$$




$$
\begin{aligned}
= & \left(\frac{T_{D 1}}{2 \lambda^{2}}\right) \int \mathrm{dt} \operatorname{Tr}\left(\Sigma_{M, N=2}^{9}\left[X^{M}, X^{N}\right]^{2}\right) \\
& -\left(\frac{T_{D 1}}{2 \lambda^{2}}\right) \int \mathrm{dt} \operatorname{Tr}\left(\Sigma_{M, N=0}^{1}\left[X^{M}, X^{N}\right]^{2}\right) \\
& -6 T_{M 0} \int \mathrm{dt} \Sigma_{M, N, L, E, F, G=0, \neq p}^{8} \varepsilon_{M N L D} \varepsilon_{E F G}^{D} \\
& \times X^{M} X^{N} X^{L} X^{E} X^{F} X^{G} \\
= & V_{\text {separation }}+V_{\text {Extra }, 2}+V_{\text {Extra }, 3}
\end{aligned}
$$

where we define

$$
\begin{aligned}
V_{\text {Extra }, 2}= & -\left(\frac{T_{D 1}}{2 \lambda^{2}}\right) \int \operatorname{dt} \operatorname{Tr}\left(\Sigma_{M, N=0}^{2}\left[X^{M}, X^{N}\right]^{2}\right), \\
V_{\text {Extra }, 3}= & -6 T_{M 0} \int \operatorname{dt} \Sigma_{M, N, L, E, F, G=0, \neq p}^{8} \\
& \times \varepsilon_{M N L D} \varepsilon_{E F G}^{D} X^{M} X^{N} X^{L} X^{E} X^{F} X^{G} .
\end{aligned}
$$

These extra energies supply the required energy for compactifying $D(p-1)$ on another circle. Following Eq. (6), we can write

$$
\begin{aligned}
& S_{D(p-1)}=S_{D(p-2)}+S_{D 1}-V_{\text {separation }} \\
& \quad \rightarrow S_{D(p-1)}+V_{\text {separation }}=S_{D(p-2)}+S_{D 1} \\
& \quad \rightarrow S_{D p}+2 V_{\text {separation }}=S_{D(p-1)}+S_{D 1}+V_{\text {separation }} \\
& \quad=S_{D(p-2)}+S_{D 1}+S_{D 1} .
\end{aligned}
$$

For example, by compactifying one $D 5$-brane on two circles, one D3-brane and two D1-branes are created:

$$
\begin{aligned}
& S_{D(p-1)}=S_{D(p-2)}+S_{D 1}-V_{\text {separation }} \\
& \quad \rightarrow S_{D(p-1)}+V_{\text {separation }}=S_{D(p-2)}+S_{D 1} \\
& \quad \rightarrow S_{D 5}+2 V_{\text {separation }}=S_{D(4)}+S_{D 1}+V_{\text {separation }} \\
& \quad=S_{D(3)}+S_{D 1}+S_{D 1} .
\end{aligned}
$$

This equation shows that the origin of the $D 3$-brane is the D5-brane. On the one hand, this D5-brane is produced by joining and growing $D 0$-branes. Also, $D 0$-branes are created by compactifying $M 0$-branes. If we assume that our universe is located on a D3-brane, we can claim that the main cause of the birth of the universe is the transition of $N M 0$-branes to a $D 3$-brane via the process $5 M 0 \rightarrow 5 D 0+$ extra energy $\rightarrow$ $D 5+$ extra energy $\rightarrow D 3+D 1+D 1$.

\section{The Padmanabhan idea in D3-D1 system}

In this section, we will construct the Padmanabhan idea in the $D 3-D 1$ system and argue that the expansion of the universe is controlled by the evolution of branes in extra dimensions. We will show that the first D1-brane which is closer to our universe dissolves in it, increases the inequality between the number of degrees of freedom on the holographic surface and inside the bulk, and leads to inflation. Then this brane gives its energy to our universe's brane, annihilates and inflation ends.
After that, the extra energies that are produced in compactifications cause an expansion and deceleration epoch. Finally, another D1-brane interacts with our universe and leads to a second phase of acceleration.

Using Eq. (6), we can obtain the relevant action for the D3- and D1-branes:

$$
\begin{aligned}
S_{D 3}= & -T_{D 3} \int \mathrm{d}^{4} \sigma \operatorname{Tr}\left(\Sigma _ { a , b = 0 } ^ { 3 } \Sigma _ { i , j = 4 } ^ { 9 } \left\{\partial_{a} X^{i} \partial_{b} X^{i}\right.\right. \\
& \left.\left.-\frac{1}{2 \lambda^{2}}\left[X^{i}, X^{j}\right]^{2}+\frac{\lambda^{2}}{4}\left(F_{a b}\right)^{2}\right\}\right), \\
S_{D 1}= & -T_{D 1} \int \mathrm{d}^{2} \sigma \operatorname{Tr}\left(\Sigma _ { a , b = 0 } ^ { 1 } \Sigma _ { i , j = 2 } ^ { 9 } \left\{\partial_{a} X^{i} \partial_{b} X^{i}\right.\right. \\
& \left.\left.-\frac{1}{2 \lambda^{2}}\left[X^{i}, X^{j}\right]^{2}+\frac{\lambda^{2}}{4}\left(F_{a b}\right)^{2}\right\}\right) .
\end{aligned}
$$

Let us now to build the Padmanabhan idea in the $D 3-D 1$ system. To this aim, we need to compute the contribution of this system to the degrees of freedom of the surface and the bulk. To this end, we write the following relations between these degrees of freedom and the energy of D1- and D3branes:

$N_{\text {sur }} \sim E_{D 3} \quad N_{\text {bulk }} \sim E_{D 3-D 1}=E_{D 3}+E_{D 1}$

$N_{\text {sur }}-N_{\text {bulk }} \simeq E_{D 1}$

where $E_{D 3}$ and $E_{D 1}$ are the energies of the D3- and D1branes, respectively. Now, we want to calculate these energies by using the action (13):

$$
\begin{aligned}
H_{D 3}= & \Sigma_{a, b=0}^{3} \Sigma_{i, j=4}^{9} \Pi_{i}\left(\partial_{t} X^{i}\right)-L_{D 3} \\
\Pi= & \frac{\partial L}{\partial\left(\partial_{t} X^{i}\right)}=-\left(\partial_{t} X^{i}\right) \\
L_{D 3}= & \operatorname{Tr}\left(\Sigma _ { a , b = 0 } ^ { 3 } \Sigma _ { i , j = 4 } ^ { 9 } \left\{\partial_{a} X^{i} \partial_{b} X^{i}\right.\right. \\
& \left.\left.-\frac{1}{2 \lambda^{2}}\left[X^{i}, X^{j}\right]^{2}+\frac{\lambda^{2}}{4}\left(F_{a b}\right)^{2}\right\}\right) \\
E_{D 3}= & -T_{D 3} \int \mathrm{d}^{4} \sigma H \\
= & -T_{D 3} \int \mathrm{d}^{4} \sigma \operatorname{Tr}\left(\Sigma _ { a , b = 0 } ^ { 3 } \Sigma _ { i , j = 4 } ^ { 9 } \left\{\partial_{a} X^{i} \partial_{b} X^{i}\right.\right. \\
& \left.\left.+\frac{1}{2 \lambda^{2}}\left[X^{i}, X^{j}\right]^{2}-\frac{\lambda^{2}}{4}\left(F_{a b}\right)^{2}\right\}\right), \\
H_{D 1}= & \Sigma_{a, b=0}^{1} \Sigma_{i, j=2}^{9} \Pi_{i}\left(\partial_{t} X^{i}\right)-L_{D 1} \\
\Pi= & \frac{\partial L}{\partial\left(\partial_{t} X^{i}\right)}=-\left(\partial_{t} X^{i}\right) \\
& \left.\left.-\frac{1}{2 \lambda^{2}}\left[X^{i}, X^{j}\right]^{2}+\frac{\lambda^{2}}{4}\left(F_{a b}\right)^{2}\right\}\right) \\
L_{D 1}= & \operatorname{Tr}\left(\Sigma _ { a , b = 0 } ^ { 1 } \Sigma _ { i , j = 2 } ^ { 9 } \left\{\partial_{a} X^{i} \partial_{b} X^{i}\right.\right. \\
\Pi^{i} &
\end{aligned}
$$




$$
\begin{aligned}
E_{D 1}= & -T_{D 1} \int \mathrm{d}^{2} \sigma H=-T_{D 1} \\
& \times \int \mathrm{d}^{2} \sigma \operatorname{Tr}\left(\Sigma _ { a , b = 0 } ^ { 1 } \Sigma _ { i , j = 2 } ^ { 9 } \left\{\partial_{a} X^{i} \partial_{b} X^{i}\right.\right. \\
& \left.\left.+\frac{1}{2 \lambda^{2}}\left[X^{i}, X^{j}\right]^{2}-\frac{\lambda^{2}}{4}\left(F_{a b}\right)^{2}\right\}\right) .
\end{aligned}
$$

Minimizing the relevant actions (13) and (14) and also the energies (16) and (17) for D3- and D1-branes yields the following condition [21]:

$\partial_{\sigma} X^{i}= \pm \frac{i}{2} \varepsilon^{i j k}\left[X^{j}, X^{k}\right]$.

The desired solution is given by

$X^{i}= \pm \frac{\alpha^{i}}{2 \sigma}$

where the $\alpha^{i}$ are an $N \times N$ representation of the $\mathrm{SU}(2)$ algebra,

$\left[\alpha^{i}, \alpha^{j}\right]=i \varepsilon^{i j k} \alpha^{k}$.

Using Eq. (18), we can obtain the minimum energy of $D 3$ and $D 1$-branes:

$$
\begin{aligned}
& E_{D 3, \min }=7 T_{D 3} \int \mathrm{d}^{4} \sigma \operatorname{Tr}\left(\Sigma_{a, b=0}^{3}\left\{\frac{\lambda^{2}}{4}\left(F_{a b}\right)^{2}\right\}\right) \\
& E_{D 1, \min }=7 T_{D 1} \int \mathrm{d}^{2} \sigma \operatorname{Tr}\left(\Sigma_{a, b=0}^{1}\left\{\frac{\lambda^{2}}{4}\left(F_{a b}\right)^{2}\right\}\right) .
\end{aligned}
$$

Following the rules in (7), we can obtain the solutions for gauge fields in $D 3$ - and $D 1$-branes:

$\lambda F_{01}$ in D1-brane $\rightarrow \partial_{t} X^{1}$ in D3-brane

$F_{a b}$ in D3-brane $\rightarrow\left[X^{a}, X^{b}\right]$ in D1-brane $\Rightarrow$

$X^{i} \sim \frac{1}{2 \sigma_{1}}, \quad A^{i} \sim \frac{1}{2 \sigma_{3}} \quad$ in D1-brane

$X^{i} \sim \frac{1}{2 \sigma_{3}}, \quad A^{i} \sim \frac{1}{2 \sigma_{1}} \quad$ in D3-brane

where $\sigma_{1}$ and $\sigma_{3}$ are coordinates of $D 1$ - and D3-branes, respectively. With increasing time, $\sigma_{1}$ decreases and is reduced to zero at the end of inflation, but $\sigma_{3}$ is increased. For this reason, we assume $\sigma_{1} \sim \frac{1}{t}$ and $\sigma_{3} \sim t$. Choosing these approximations needs some further discussion and explanations. According to this model, the universe is located on the D3-brane; thus, due to the time evolution and the universe's expansion, the $D 3$-brane expands and $\sigma_{3}$, which is the coordinate of the $D 3$-brane, grows and has a direct relation with time. On the other hand, the main cause of inflation is dissolving of the D1-brane into the D3-brane which represents our universe. Therefore, by passing time and the universe's inflation, $\sigma_{1}$, which is the coordinate of the $D 1$-brane, decreases and thus it is related with the inverse of time. Furthermore, due to the evolution in time and the disappearing of the $D 1$ brane, gauge fields which are stuck to it have to vanish. As can be seen from Eq. (22), the gauge field on the D1-brane is related to $\frac{1}{\sigma_{3}}$ and thus by evolving time and disappearing $D 1$-brane and the gauge field, $\sigma_{3}$ increases.

With this assumption and using Eqs. (16), (17), and (22), and also condition in (18), we can calculate the energy of the $D 1$ - and D3-branes and the number of degrees of freedom on the holographic surface and one in the bulk:

$$
\begin{aligned}
N_{\text {sur }}- & N_{\text {bulk }} \simeq E_{D 1} \simeq 14 \pi^{2} l_{s}^{4} T_{D 1}\left[\frac{t_{\text {inf }}-t}{t_{\text {inf }}}\right] \\
N_{\text {sur }} \simeq & E_{D 3}+\left(E_{D 1, \text { inf }}-E_{D 1}\right) \\
\simeq & 15 \pi^{2} l_{s}^{4} T_{D 3}\left[\frac{t^{5}}{60}+\frac{t_{\text {inf }} t^{4}}{12}-\frac{t_{\text {inf }}^{2} t^{3}}{9}-\frac{t_{\text {inf }}^{3} t^{2}}{24}\right. \\
& \left.+\frac{t_{\text {inf }}^{4} t}{2}+14 \pi^{2} l_{s}^{4} T_{D 1}\left[\frac{t}{t_{\text {inf }}}\right]\right]
\end{aligned}
$$

where $\left(E_{D 1 \text {,inf }}=14 \pi^{2} l_{s}^{4} T_{D 1}\right)$ is the energy of the $D 1$-brane at the beginning of inflation, $\left(E_{D 1 \text {,inf }}-E_{D 1}\right)$ is the amount of energy which dissolves in the $D 3$-brane, and $t=t_{\text {inf }}$ is the time of the end of inflation. As can be seen from this equation, the difference between the number of degrees of freedom on the holographic surface and the bulk decreases with time and shrinks to zero at the end of inflation $\left(t=t_{\text {inf }} \Rightarrow N_{\text {sur }}=\right.$ $\left.N_{\text {bulk }}\right)$. This means that our calculations are consistent with the Padmanabhan idea and thus our model works.

These equations help us to obtain the relation between some of the cosmological parameters like the deceleration parameter and the evolution of the $D 1$ - and $D 3$-branes. To this end, first, we calculate the Hubble parameter via the following equation:

$$
\begin{aligned}
N_{\text {sur }} & =\frac{4 \pi r_{A}^{2}}{l_{p}^{2}} \quad r_{A}=\frac{1}{H} \Rightarrow \\
N_{\text {sur }} & =\frac{4 \pi}{\left(l_{p} H\right)^{2}} \simeq 15 \pi^{2} l_{s}^{4} T_{D 3}\left[\frac{t^{5}}{60}+\frac{t_{\text {inf }} t^{4}}{12}\right. \\
& \left.-\frac{t_{\text {inf }}^{2} t^{3}}{9}-\frac{t_{\text {inf }}^{3} t^{2}}{24}+\frac{t_{\text {inf }}^{4} t}{2}+14 \pi^{2} l_{s}^{4} T_{D 1}\left[\frac{t}{t_{\text {inf }}}\right]\right] \Rightarrow \\
H= & \frac{2}{\sqrt{15 \pi l_{p}^{2} l_{s}^{4} T_{D 3}\left[\frac{t^{5}}{60}+\frac{\text { tint }^{4} t^{4}}{12}-\frac{t_{\text {int }}^{2} t^{3}}{9}-\frac{t_{\text {inf }}^{3} t^{2}}{24}+\frac{t_{\text {inf }}^{4}}{2}\right]+14 \pi^{2} l_{s}^{4} T_{D 1}\left[\frac{t}{t_{\text {inf }}}\right]}}
\end{aligned}
$$

where $H$ is the Hubble parameter and $l_{p}$ is the Planck length. Using this equation, we can obtain the deceleration parameter during the inflation era in terms of time:

$$
\begin{aligned}
q= & \frac{d}{\mathrm{dt}}\left(\frac{1}{H}\right)-1 \simeq \sqrt{15 \pi l_{p}^{2} l_{s}^{4} T_{D 3} t_{\mathrm{inf}}^{4}}\left[\frac{t^{4}}{24 t_{\mathrm{inf}}^{4}}+\frac{t^{3}}{6 t_{\mathrm{inf}}^{3}}\right. \\
& \left.-\frac{t^{2}}{6 t_{\mathrm{inf}}^{2}}-\frac{t}{24 t_{\mathrm{inf}}}\right]-14 \pi^{2} l_{s}^{4} T_{D 1} t\left[1-\frac{t}{t_{\mathrm{inf}}}\right] .
\end{aligned}
$$

This equation indicates that while the age of the universe $(t)$ increases, the deceleration parameter reduces to lower 


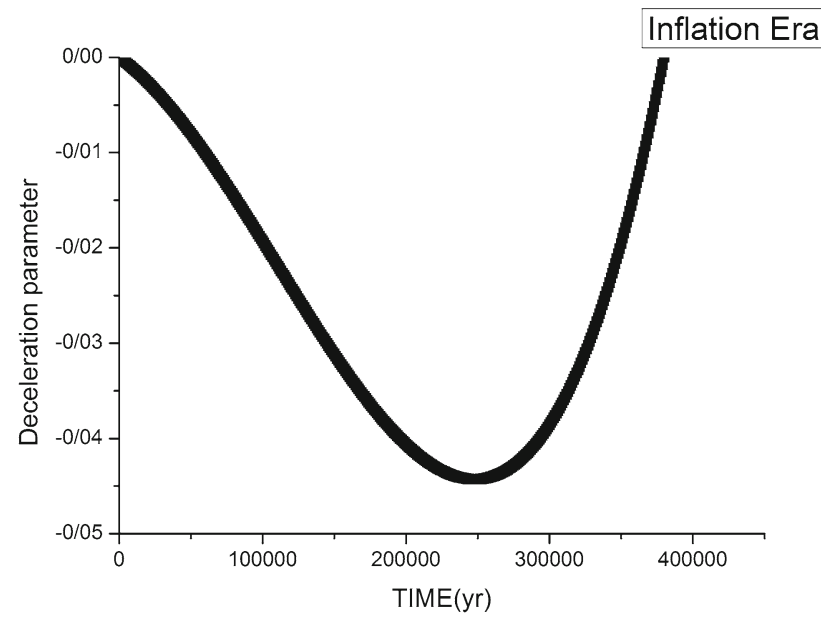

Fig. 1 The deceleration parameter for the inflation era of the expansion history as a function of the $t$ where $t$ is the age of universe. In this plot, we choose $t_{\text {inf }}=380000, T_{D 3}=10000$ and $l_{s}=0.1$

negative values, turns over a minimum, increases and tends to zero at $t=t_{\text {inf }}$ (see Fig. 1). This means that the $D 1$-brane has disappeared at the end of inflation; however, the rate of acceleration of the universe is increased very fast and tends to large values in this epoch.

With the disappearance of the D1-brane, inflation ends and a deceleration epoch begins. At this stage, extra energies that are produced in previous compactifications of branes cause an expansion of the universe. Some of these energies are introduced in Eq. (10). Applying $V_{\text {Extra,2 }}=$ $\left(\frac{T_{D 1}}{2 \lambda^{2}}\right) \int \operatorname{dt} \operatorname{Tr}\left(\Sigma_{M, N=0}^{2}\left[X^{M}, X^{N}\right]^{2}\right)$ in this equation, assuming $\sigma_{\text {extra }} \sim \frac{1}{t}$ and $\sigma_{3} \sim t$, and using Eqs. (13), (22), and (18), we can obtain the amount of extra energy and also the energy of the $D 3$-branes and the number of degrees of freedom on the holographic surface and one in the bulk region:

$$
\begin{aligned}
N_{\text {sur }} & -N_{\text {bulk }} \simeq E_{\text {extra,inf }-t} \simeq-\left(\frac{T_{D 1}}{2 \lambda^{2}}\right) \\
& \times \int \mathrm{dt} \operatorname{Tr}\left(\Sigma_{M, N=0}^{2}\left[X^{M}, X^{N}\right]^{2}\right) \\
\simeq & \left(\frac{T_{D 1}}{4 \pi^{2} l_{s}^{4}}\right)\left[\frac{\left(t_{\mathrm{dec}}-t_{\mathrm{inf}}\right)^{3}-\left(t-t_{\mathrm{inf}}\right)^{3}}{\left(t-t_{\mathrm{inf}}\right)^{3}}\right], \\
N_{\mathrm{sur}} \simeq E_{D 3} \simeq E_{D 3, \mathrm{inf}}+\left(E_{\mathrm{extra}, \mathrm{inf}-\mathrm{dec}}-E_{\mathrm{extra}, \mathrm{inf}-t)}\right. & \quad+5 T_{D 3} \int \mathrm{d}^{4} \sigma \operatorname{Tr}\left(\Sigma_{a=0}^{3}\left\{\frac{\lambda^{2}}{4}\left(\partial_{a}\left(\frac{1}{\sigma_{1}}\right)\right)^{2}\right\}\right) \\
\simeq & E_{D 3, \text { inf }}+15 \pi^{2} l_{s}^{4} T_{D 3}\left[\frac{\left(t_{\mathrm{dec}}-t\right)^{5}}{60}\right. \\
+ & \frac{\left(t_{\mathrm{dec}}-t_{\mathrm{inf}}\right)\left(t_{\mathrm{dec}}-t\right)^{4}}{12}-\frac{\left(t_{\mathrm{dec}}-t_{\mathrm{inf}}\right)^{2}\left(t_{\mathrm{dec}}-t\right)^{3}}{9} \\
& \left.-\frac{\left(t_{\mathrm{dec}}-t_{\mathrm{inf}}\right)^{3}\left(t_{\mathrm{dec}}-t\right)^{2}}{24}+\frac{\left(t_{\mathrm{dec}}-t_{\mathrm{inf}}\right)^{4}\left(t_{\mathrm{dec}}-t\right)}{2}\right] \\
& +\left(\frac{T_{D 1}}{4 \pi^{2} l_{s}^{4}}\right)\left[\frac{\left(t_{\mathrm{dec}}-t_{\mathrm{inf}}\right)^{3}}{\left(t-t_{\mathrm{inf}}\right)^{3}}\right]
\end{aligned}
$$

where $E_{D 3 \text {,inf }}=\frac{480 \pi^{2} l_{s}^{4} T_{D 3} t_{\text {inf }}^{5}}{72}$ is the energy of the D3-brane at the end of inflation, $\left(E_{\text {extra,inf-dec }}-E_{\text {extra,inf }-t}\right)$ is the amount of energy that dissolves in the D3-brane during the deceleration era and $t=t_{\mathrm{dec}}$ is the age of the universe at the end of the deceleration epoch. Similar to the inflation era, the difference between the number of degrees of freedom on the holographic surface and the bulk decreases with time and shrinks to zero at the end of deceleration $\left(t=t_{\mathrm{dec}} \Rightarrow\right.$ $\left.N_{\text {sur }}=N_{\text {bulk }}\right)$. The Hubble parameter during this era can be calculated as

$$
\begin{aligned}
N_{\mathrm{sur}}= & \frac{4 \pi r_{A}^{2}}{l_{p}^{2}} \quad r_{A}=\frac{1}{H} \Rightarrow \\
N_{\mathrm{sur}}= & \frac{4 \pi}{\left(l_{p} H\right)^{2}} \simeq E_{D 3, \mathrm{inf}}+15 \pi^{2} l_{S}^{4} T_{D 3}\left[\frac{\left(t_{\mathrm{dec}}-t\right)^{5}}{60}\right. \\
& +\frac{\left(t_{\mathrm{dec}}-t_{\mathrm{inf}}\right)\left(t_{\mathrm{dec}}-t\right)^{4}}{12}-\frac{\left(t_{\mathrm{dec}}-t_{\mathrm{inf}}\right)^{2}\left(t_{\mathrm{dec}}-t\right)^{3}}{9} \\
& \left.-\frac{\left(t_{\mathrm{dec}}-t_{\mathrm{inf}}\right)^{3}\left(t_{\mathrm{dec}}-t\right)^{2}}{24}+\frac{\left(t_{\mathrm{dec}}-t_{\mathrm{inf}}\right)^{4}\left(t_{\mathrm{dec}}-t\right)}{2}\right] \\
& +\left(\frac{T_{D 1}}{4 \pi^{2} l_{s}^{4}}\right)\left[\frac{\left(t_{\mathrm{dec}}-t_{\mathrm{inf}}\right)^{3}}{\left(t-t_{\mathrm{inf}}\right)^{3}}\right] \Rightarrow \\
H= & \frac{4 \pi}{l_{p} \sqrt{N_{\mathrm{sur}}}} .
\end{aligned}
$$

With the help of this equation, we can derive the deceleration parameter during the deceleration epoch in terms of time:

$$
\begin{aligned}
q= & \frac{d}{\mathrm{dt}}\left(\frac{1}{H}\right)-1 \simeq \sqrt{15 \pi l_{p}^{2} l_{s}^{4} T_{D 3}\left(t_{\mathrm{dec}}-t_{\mathrm{inf}}\right)^{4}} \\
& \times\left[-\frac{\left(t_{\mathrm{dec}}-t\right)^{4}}{24\left(t_{\mathrm{dec}}-t_{\mathrm{inf}}\right)^{4}}-\frac{\left(t_{\mathrm{dec}}-t\right)^{3}}{6\left(t_{\mathrm{dec}}-t_{\mathrm{inf}}\right)^{3}}\right. \\
& \left.+\frac{\left(t_{\mathrm{dec}}-t\right)^{2}}{6\left(t_{\mathrm{dec}}-t_{\mathrm{inf}}\right)^{2}}+\frac{\left(t_{\mathrm{dec}}-t\right)}{24\left(t_{\mathrm{dec}}-t_{\mathrm{inf}}\right)}\right] \\
& +\left(\frac{T_{D 1}\left(t-t_{\mathrm{inf}}\right)^{2}}{4 \pi^{2} l_{s}^{4}}\right)\left[1-\left[\frac{\left(t-t_{\mathrm{inf}}\right)^{2}}{\left(t_{\mathrm{dec}}-t_{\mathrm{inf}}\right)^{2}}\right]\right] .
\end{aligned}
$$

In Fig. 2, we present the deceleration parameter in terms of time. As can be seen from this figure, the deceleration parameter increases to higher positive values, turns over a maximum, decreases, and tends to zero at the end of the deceleration epoch. Thus our model is consistent with previous predictions for the deceleration era.

After a period of time, the second $D 1$-brane become close to our universe D3-brane, dissolves in it, and leads to the present phase of acceleration. Similar to previous epochs, we assume that $\sigma_{1} \sim \frac{1}{t}$ and $\sigma_{3} \sim t$, and we use Eqs. (13), (14), and (22) and also the condition in (18), to calculate the energy of the $D 1$ - and $D 3$-branes and the number of degrees of freedom on the holographic surface and bulk: 


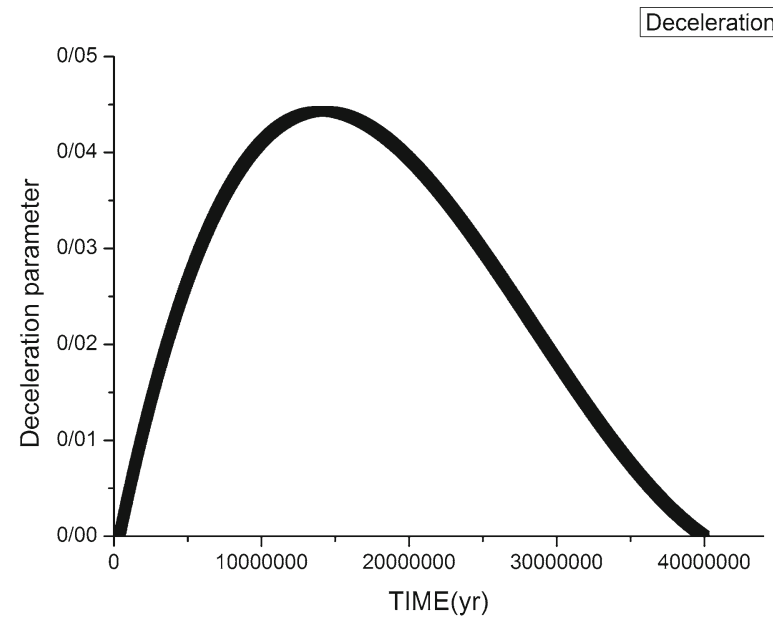

Fig. 2 The deceleration parameter for the deceleration era of the expansion history as a function of $t$ where $t$ is the age of the universe. In this plot, we choose $t_{\text {inf }}=380000, t_{\mathrm{dec}}=40000000$ years, $T_{D 3}=10000$, and $l_{s}=0.1$

$$
\begin{aligned}
& N_{\text {sur }}-N_{\text {bulk }} \simeq E_{D 1, t-t_{\text {dec }}} \simeq 14 \pi^{2} l_{s}^{4} \\
& \times T_{D 1}\left[\frac{\left(t_{a c}-t_{\mathrm{dec}}\right)-\left(t-t_{\mathrm{dec}}\right)}{\left(t_{a c}-t_{\mathrm{dec}}\right)}\right], \\
& N_{\text {sur }} \simeq E_{D 3} \simeq E_{D 3, \text { inf }}+E_{D 3, \mathrm{dec}} \\
& +\left(E_{D 1, t_{a c}-t_{\mathrm{dec}}}-E_{D 1, t-t_{\mathrm{dec}}}\right) \\
& +5 T_{D 3} \int \mathrm{d}^{4} \sigma \operatorname{Tr}\left(\Sigma_{a=0}^{3}\left\{\frac{\lambda^{2}}{4}\left(\partial_{a}\left(\frac{1}{\sigma_{1}}\right)\right)^{2}\right\}\right) \\
& \simeq E_{D 3, \text { inf }}+E_{D 3, \mathrm{dec}}+15 \pi^{2} l_{s}^{4} T_{D 3} \\
& \times\left[\frac{\left(t_{a c}-t\right)^{5}}{60}+\frac{\left(t_{a c}-t_{\mathrm{dec}}\right)\left(t_{a c}-t\right)^{4}}{12}\right. \\
& -\frac{\left(t_{a c}-t_{a c}\right)^{2}\left(t_{a c}-t\right)^{3}}{9} \\
& -\frac{\left(t_{a c}-t_{\mathrm{dec}}\right)^{3}\left(t_{a c}-t\right)^{2}}{24} \\
& \left.+\frac{\left(t_{a c}-t_{\mathrm{dec}}\right)^{4}\left(t_{a c}-t\right)}{2}\right] \\
& -14 \pi^{2} l_{s}^{4} T_{D 1}\left[\frac{\left(t-t_{\mathrm{dec}}\right)}{\left(t_{a c}-t_{\mathrm{dec}}\right)}\right] \text {. }
\end{aligned}
$$

In the above equation, $E_{D 3, \mathrm{dec}}=\frac{480 \pi^{2} l_{s}^{4} T_{D 3}\left(t_{\mathrm{dec}}-t_{\mathrm{inf}}\right)^{5}}{72}$ is the amount of energy that the $D 3$-brane acquires during the deceleration era, $\left(E_{D 1, t_{a c}-t_{\mathrm{dec}}}-E_{D 1, t-t_{\mathrm{dec}}}\right)$ is the amount of energy of the $D 1$-brane that dissolves in the $D 3$-brane during late-time acceleration, and $t=t_{a c}$ is the age of the universe at the end of the present acceleration epoch. Similar to previous epochs, the difference between the number of degrees of freedom on the holographic surface and bulk decreases with time and shrinks to zero at the end of the late-time acceleration ( $t=t_{a c} \Rightarrow N_{\text {sur }}=N_{\text {bulk }}$ ). The Hubble parameter during this era can be obtained as

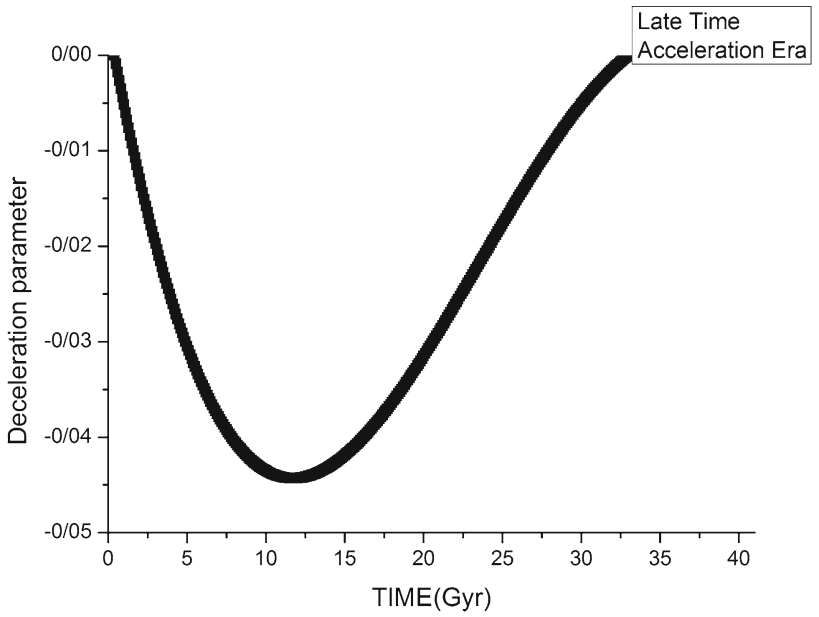

Fig. 3 The deceleration parameter for the acceleration era of the expansion history as a function of $t$, where $t$ is the age of universe. In this plot, we choose $t_{a c}=33 \mathrm{Gyr}, t_{\mathrm{dec}}=0.4 \mathrm{Gyr}, T_{D 3}=10000$, and $l_{s}=0.1$

$$
\begin{aligned}
N_{\text {sur }}= & \frac{4 \pi r_{A}^{2}}{l_{p}^{2}} \quad r_{A}=\frac{1}{H} \Rightarrow \\
N_{\text {sur }}= & \frac{4 \pi}{\left(l_{p} H\right)^{2}} \simeq E_{D 3, \text { inf }}+E_{D 3, \mathrm{dec}} \\
& +15 \pi^{2} l_{s}^{4} T_{D 3}\left[\frac{\left(t_{a c}-t\right)^{5}}{60}+\frac{\left(t_{a c}-t_{\mathrm{dec}}\right)\left(t_{a c}-t\right)^{4}}{12}\right. \\
& -\frac{\left(t_{a c}-t_{a c}\right)^{2}\left(t_{a c}-t\right)^{3}}{9}-\frac{\left(t_{a c}-t_{\mathrm{dec}}\right)^{3}\left(t_{a c}-t\right)^{2}}{24} \\
& \left.+\frac{\left(t_{a c}-t_{\mathrm{dec}}\right)^{4}\left(t_{a c}-t\right)}{2}\right]-14 \pi^{2} l_{s}^{4} T_{D 1}\left[\frac{\left(t-t_{\mathrm{dec}}\right)}{\left(t_{a c}-t_{\mathrm{dec}}\right)}\right] \Rightarrow \\
H= & \frac{4 \pi}{l_{p} \sqrt{N_{\mathrm{sur}}}} .
\end{aligned}
$$

Using this equation, we can calculate the deceleration parameter during the present acceleration epoch in terms of time:

$$
\begin{aligned}
q= & \frac{d}{\mathrm{dt}}\left(\frac{1}{H}\right)-1 \simeq \sqrt{15 \pi l_{p}^{2} l_{s}^{4} T_{D 3}\left(t_{a c}-t_{\mathrm{dec}}\right)^{4}} \\
& \times\left[\frac{\left(t_{a c}-t\right)^{4}}{24\left(t_{a c}-t_{\mathrm{dec}}\right)^{4}}+\frac{\left(t_{a c}-t\right)^{3}}{6\left(t_{a c}-t_{\mathrm{dec}}\right)^{3}}\right. \\
& \left.-\frac{\left(t_{a c}-t\right)^{2}}{6\left(t_{a c}-t_{\mathrm{dec}}\right)^{2}}-\frac{\left(t_{a c}-t\right)}{24\left(t_{\mathrm{dec}}-t_{\mathrm{inf}}\right)}\right] \\
& -14 \pi^{2} l_{s}^{4} T_{D 1}\left(t-t_{\mathrm{dec}}\right)\left[1-\frac{\left(t-t_{\mathrm{dec}}\right)}{\left(t_{a c}-t_{\mathrm{dec}}\right)}\right] .
\end{aligned}
$$

In Fig. 3, we show the deceleration parameter during the late time acceleration era in terms of time. It is clear that the deceleration parameter is negative at the present stage, which is a signature of the acceleration. This result is in agreement with recent experimental data and thus our model works. 


\section{Summary and discussion}

We constructed the Padmanabhan idea in $M$-theory and argued that the birth and expansion of the universe are controlled by the evolution of branes in extra dimensions. To this end, first, we obtained the relevant action for $N M 0$-branes by replacing the Nambu-Poisson structure of two dimensional manifolds in $D$-branes by the structure of a three dimensional one. At the second stage, we compactified them on one circle and derived the action for $N D 0$-branes. We showed that $N$ $D 0$-branes join to each other, grow, and form a D5-brane. Then we compactified this brane on two circles and obtained the action for our universe's brane, two $D 1$-branes and some extra energies that are created due to this compactifications. Next, we discussed that one of the $D 1$-branes, which is closer to our universe's brane, dissolves in it, leads to an increase in the difference between the number of degrees of freedom on the holographic surface and in the bulk region and the occurrence of an inflation era. After a short time, this $D 1$ brane annihilates, the numbers of degrees of freedom on the boundary surface and bulk region become equal, inflation ends, and a deceleration epoch begins. During this era, extra energies that are produced due to compactification are the main causes of expansion. Finally, we argued that the interaction of another $D 1$-brane with our universe's brane leads to an inequality between the degrees of freedom and the occurrence of a new phase of acceleration.

Acknowledgments The work of Alireza Sepehri has been supported financially by Research Institute for Astronomy and Astrophysics of Maragha (RIAAM), Iran, under research Project No. 1/4165-13. We thank the referee for nice comments that help us to improve our paper.

Open Access This article is distributed under the terms of the Creative Commons Attribution 4.0 International License (http://creativecomm ons.org/licenses/by/4.0/), which permits unrestricted use, distribution, and reproduction in any medium, provided you give appropriate credit to the original author(s) and the source, provide a link to the Creative Commons license, and indicate if changes were made.

Funded by SCOAP ${ }^{3}$.

\section{References}

1. T. Padmanabhan. arXiv:1206.4916 [hep-th]

2. K. Yang, Y.-X. Liu, Y.-Q. Wang, Phys. Rev. D 86, 104013 (2012)

3. Y. Ling, W.-J. Pan, Phys. Rev. D 88, 043518 (2013)

4. A. Sheykhi, Phys. Rev. D 87, 061501(R) (2013)

5. M. Eune, W. Kim, Phys. Rev. D 88, 067303 (2013)

6. E. Chang-Young, D. Lee, J. High Energy Phys. 1404, 125 (2014)

7. A.F. Ali, Phys. Lett. B 732, 335 (2014)

8. A. Sepehri et al., Phys. Lett. B 741, 92-96 (2015)

9. A. Sepehri et al., Phys. Lett. B 747, 1-8 (2015)

10. M.R. Setare, A. Sepehri, JHEP 1503, 079 (2015)

11. G. Grignani, T. Harmark, A. Marini, N.A. Obers, M. Orselli, JHEP 1106, 058 (2011)

12. M.R. Setare, A. Sepehri, Phys. Rev. D 91, 063523 (2015)

13. A. Sepehri, Phys. Lett. B 748, 328335 (2015). arXiv:1508.01407 [gr-qc]

14. J. Bagger, N. Lambert, Modeling multiple M2s. Phys. Rev. D 75, 045020 (2007). arXiv:hep-th/0611108

15. J. Bagger, N. Lambert, Gauge symmetry and supersymmetry of multiple M2-branes. Phys. Rev. D 77, 065008 (2008). arXiv:0711.0955 [hep-th]

16. J. Bagger, N. Lambert, Comments on multiple M2-branes. JHEP 0802, 105 (2008). arXiv:0712.3738 [hep-th]

17. A. Gustavsson, Algebraic structures on parallel M2-branes. arXiv:0709.1260 [hep-th]

18. A. Gustavsson, Selfdual strings and loop space Nahm equations. arXiv:0802.3456 [hep-th]

19. P.-M. Ho, Y. Matsuo, JHEP 0806, 105 (2008)

20. R.C. Myers, JHEP 9912, 022 (1999). arXiv:hep-th/9910053

21. N.R. Constable, R.C. Myers, O. Tafjord, JHEP 0106, 023 (2001)

22. A.A. Tseytlin. arXiv:hep-th/9908105

23. C.-S. Chu, D.J. Smith, JHEP 0904, 097 (2009)

24. B. Sathiapalan, Nilanjan Sircar. JHEP 0808, 019 (2008)

25. N.R. Constable, R.C. Myers, O. Tafjord, Phys. Rev. D 61, 106009 (2000)

26. J. Kluson, JHEP 0011, 016 (2000)

27. S. Mukhi, C. Papageorgakis, JHEP 0805, 085 (2008)

28. A. Sepehri, Mod. Phys. Lett. A. doi:10.1142/S0217732316500048. arXiv:1510.07961v1 [physics.gen-ph] 